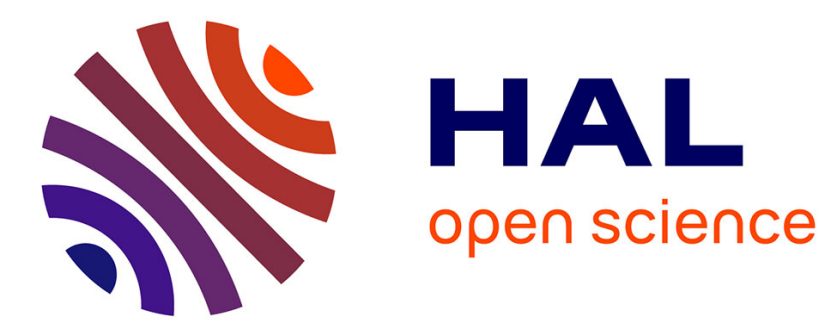

\title{
IST-06 - Réémergence de la syphilis chez les personnes infectées par le VIH en Martinique en 2015
}

\author{
Alain Putot, Benoit Rozé, Sandrine Pierre-François, M. Pircher, R. Vilain, C. \\ Miossec, N. Desbois, Patrick Hochedez, Sylvie Abel, André Cabié
}

\section{- To cite this version:}

Alain Putot, Benoit Rozé, Sandrine Pierre-François, M. Pircher, R. Vilain, et al.. IST-06 - Réémergence de la syphilis chez les personnes infectées par le VIH en Martinique en 2015. 17es Journées Nationales d'Infectiologie, Jun 2016, Lille, France. Médecine et maladies infectieuses 46 (2016) 73-74, 46 (4), pp.73 - 74 . inserm-01413481

\section{HAL Id: inserm-01413481 https://www.hal.inserm.fr/inserm-01413481}

Submitted on 9 Dec 2016

HAL is a multi-disciplinary open access archive for the deposit and dissemination of scientific research documents, whether they are published or not. The documents may come from teaching and research institutions in France or abroad, or from public or private research centers.
L'archive ouverte pluridisciplinaire HAL, est destinée au dépôt et à la diffusion de documents scientifiques de niveau recherche, publiés ou non, émanant des établissements d'enseignement et de recherche français ou étrangers, des laboratoires publics ou privés. 


\section{IST-03}

Infections sexuellement transmissibles chez la femme à La Réunion, place de Mycoplasma genitalium

R. Manaquin (1), F. Pagès (2), M. Jaffar-Bandjee (3), B. Roquebert (3), M. Moiton (3), G. Camuset (1), G. Borgherini (1), P. Poubeau (1), C. Cazanave (4)

(1) CHU Sud Réunion, Saint-Pierre,

(2) CIRE Océan Indien, Saint-Denis,

(3) CHU Félix Guyon, Saint-Denis,

(4) $\mathrm{CHU}$ de Bordeaux.

Introduction Peu de données existent sur les IST à La Réunion. Mycoplasma genitalium $(\mathrm{Mg})$, pathogène émergent responsable d'infections urogénitales, n'est pas documenté localement. L'objectif principal de cette étude était de mettre en évidence la place de $\mathrm{Mg}$ dans les infections génitales de la femme à La Réunion. Les objectifs secondaires étaient d'évaluer la prévalence de $\mathrm{Mg}$, Chlamydia trachomatis $(\mathrm{Ct})$ et Neisseria gonorrhoeae $(\mathrm{Ng})$ au sein d'une population de femmes symptomatiques à haut risque d'IST.

Matériels et méthodes Étude descriptive, rétrospective, monocentrique, à partir de prélèvements génitaux ou urinaires collectés d'avril 2011 à avril 2014 chez des femmes âgées de 14 à 39 ans. Il s'agissait d'une population à haut risque d'IST : femmes jeunes venues consulter en urgence au service de gynécologie et pour lesquelles une recherche d'IST a été faite. Chez ces femmes la recherche de $\mathrm{Ct}, \mathrm{Mg}$ et de $\mathrm{Ng}$ a été réalisée par biologie moléculaire. Les données cliniques ont été recueillies à l'aide d'une grille de lecture des dossiers.

Résultats Deux cent quatre-vingt-sept patientes ont été analysées. Une PCR multiplexe $\mathrm{Ng}$ et $\mathrm{Mg}$ a été développée. Dix prélèvements sont revenus positifs à $\mathrm{Mg}$. Les taux de prévalence de $\mathrm{Mg}$, $\mathrm{Ct}$ et $\mathrm{Ng}$ étaient respectivement de 3,5\% $\left(\mathrm{IC}_{95 \%}=[1,4-5,6]\right), 19,5 \%\left(\mathrm{IC}_{95} \%=[14,9-24,1]\right)$ et $7,7 \%\left(\mathrm{IC}_{95 \%}=[4,6-10,7]\right)$. Trois patientes étaient co-infectées aux trois bactéries, trois présentaient une coinfection à $\mathrm{Mg}$ et $\mathrm{Ct}$ et une à $\mathrm{Mg}$ et $\mathrm{Ng}$. Le célibat et la grossesse étaient significativement liés au risque d'infection à $\mathrm{Ct}$ avec des risques relatifs (RR) respectifs à $1,9[1,2-2,2]$ et $1,8[1,1-2,9]$. L'âge inférieur ou égal à 25 ans était significativement associé aux infections à $\mathrm{Ct}$ et $\mathrm{Ng}$ (RR respectifs : 3,7 [1,9-7,8] et 4,0 [1,1-11,6]). Chez les 14-25 ans, la prévalence de $\mathrm{Mg}$, Ct et $\mathrm{Ng}$ était respectivement de 4,6\% $\left(\mathrm{IC}_{95 \%}=[1,3-7,9]\right), 29,8 \%\left(\mathrm{IC}_{95 \%}=[22,5-37,1]\right)$ et $11,9 \%\left(\mathrm{IC}_{95 \%}=[6,7-17,1]\right)$.

Conclusion Cette étude met en évidence pour la première fois l'implication de Mg comme agent d'IST à La Réunion. Même si la prévalence reste faible au sein d'une population à haut risque la présence de $\mathrm{Mg}$ ainsi que les taux d'infection élevés à $\mathrm{Ng}$ et $\mathrm{Ct}$, notamment chez les 14-25 ans, soulignent l'importance des IST à La Réunion et la nécessité de relancer les programmes de santé sexuelle afin de diminuer les conséquences à long terme sur la santé des femmes.

Aucun lien d'intérêt

\section{IST-04}

Impact du Mycoplasma genitalium dans un CDAG d'Outre Mer à la lumière des autres maladies sexuellement transmissibles : étude transversale

A. Bertolotti (1), P. Gerardin (2), N. Zemali (2), R. Manaquin (2), G. Borgherini (2), A. Foucher (2), S. Picot (2), J. Jaubert (2), P. Poubeau (2), G. Camuset (2)

(1) Université de Bordeaux,

(2) $\mathrm{CHU}$ de la Réunion.

Introduction Une recrudescence des infections sexuellement transmissibles (IST) est constatée sur le territoire métropolitain, mais peu documentée en outre-mer. Nous avons mené une étude transversale dans notre centre de dépistage anonyme et gratuit (CDAG) pour estimer la prévalence des différentes IST dont celle à Mycoplasma genitalium $(\mathrm{MG})$ et rechercher les facteurs de risque associés à ce pathogène.

Matériels et méthodes Une étude transversale, monocentrique, a été menée de juin 2014 à août 2015, chez tous les patients consultant dans notre CDAG. Une PCR triplex recherchant Chlamydia trachomatis (CT), Neisseria Gonorrheae (NG) et $\mathrm{MG}$, à partir des urines chez les hommes, d'auto-écouvillon vaginal chez les femmes et d'écouvillons pharyngés et anaux chez les patients identifiés à risque était réalisé systématiquement, associée aux sérologies des IST. Les variables recueillies par un auto-questionnaire anonyme en première consultation ont été analysées par régressions logistiques.

Résultats L'étude portait sur 367 femmes (43,2\%) et 482 hommes $(56,8 \%)$, jeunes (âges médians respectifs de 26 et 30 ans), dont 51 parmi ces derniers avaient des relations homosexuelles. Des écouvillons pharyngés et rectaux étaient réalisés chez 628 et 146 patients, respectivement. La prévalence du VIH était de $0,1 \%$; celle de la syphilis de $0,8 \%$; celles de l'hépatite $\mathrm{B}$ et l'hépatite $\mathrm{C}$ de $0,4 \%$; celle du CT était de $6,4 \%, 1,9 \%$ et $6,8 \%$, respectivement en uro-génital, pharyngé et rectal; celle du NG de $0,7 \%$, $1,4 \%$ et $2,7 \%$, respectivement ; celle du MG de $1,9 \%, 0,3 \%$ et $2,0 \%$, respectivement. En analyse multivariée, les seuls facteurs identifiés comme associés au MG urogénital étaient un portage concomitant de CT urinaire (OR 7,0; $\mathrm{p}<0,01)$, la présence d'anticorps anti-Hbc (OR 8,2; $<<0,01)$, un antécédent de syphilis (OR 19,3; $\mathrm{p}=0,02)$ et l'absence de partenaire de sexe différent (OR 5,3; $\mathrm{p}=0,02)$. Seul un antécédent d'infection par le gonocoque était associé à la positivité du MG rectal (OR 74,6; p < 0,01).

Conclusion La prévalence des IST reste importante au sein de la population consultant en CDAG dans notre DOM. Le risque d'infection par MG étant difficile à apprécier lors de l'interrogatoire, nous proposons de l'authentifier par une recherche systématique par PCR multiplex.

Aucun lien d'intérêt

\section{IST-05}

\section{Un spirochète peut en cacher un autre}

O. Cannesson (1), R. Hecquette Ruz (1), A. Filali (1), A. Mambie (2), D. Poitrenaud (3), A. Leguern (4), E. Faure (1), M. Douaud (1), K. Faure (1), B. Guery (1)

(1) CHRU Lille, Maladies Infectieuses,

(2) CHR de Douai,

(3) CHR de Béthune

(4) CHR de Saint-Vincent.

Introduction L'augmentation de l'incidence de la syphilis en France peut mettre le praticien dans une situation un peu particulière.

Matériels et méthodes En effet, lors d'un bilan systématique chez un patient présentant une choriorétinite, la sérologie Syphilis s'est révélée positive, dans le sang et le liquide cérébro-spinale (LCS). S'en est suivie une ponction lombaire, elle aussi positive, et un le traitement par pénicilline $\mathrm{G}$ est débuté immédiatement.

La sérologie Lyme est elle aussi positive, mais plus tardive, confirmée par un Western Blot complet, dans le sang et le LCS. S'en est suivi un débat : quel est le spirochète responsable de la symptomatologie ? En effet, dans la littérature, les deux bactéries peuvent être responsables de ce genre de tableau. Ne serait-ce pas une réaction croisée ?

Nous nous sommes attardés sur les facteurs de risque du patient : il s'agit d'un patient ayant des relations sexuelles non protégées avec d'autres hommes, mais il est aussi amateur de cueillette de champignons dans les bois avoisinants et a déjà présenté une symptomatologie compatible avec un érythème migrant.

Résultats $\mathrm{Ne}$ pouvant pas faire la part des choses, le patient a bénéficié d'un traitement par Pénicilline $\mathrm{G}$ intraveineuse pendant 14 jours puis 10 jours de Ceftriaxone. Le patient a pratiquement récupéré l'entièreté de son acuité visuelle à un an de l'épisode.

Conclusion La littérature ne permet d'affirmer s'il s'agit d'une coinfection ou d'un faux positif pour la Syphilis ou la maladie de Lyme. Les avis d'experts seraient plus en faveur d'un faux positif pour la Syphilis. Vue l'incidence en constante augmentation de la Syphilis, le cas pourrait se présenter fréquemment, d'où la nécessité d'une étude prospective permettant d'évaluer sur un grand échantillon la probabilité d'une coïnfection ou de faux positif lors d'une infection à spirochète.

Aucun lien d'intérêt

\section{IST-06}

Réémergence de la syphilis chez les personnes infectées par le VIH en Martinique en 2015

A. Putot, B. Rozé, S. Pierre-François, M. Pircher, R. Vilain, C. Miossec, N. Desbois, P. Hochedez, S. Abel, A. Cabié

CHU de Martinique.

Introduction En 2004 a débuté une épidémie de syphilis en Martinique chez les hommes ayant des rapports sexuels avec des hommes (HSH) et vivant avec le VIH, pour se généraliser secondairement à l'ensemble des groupes à 
risque. L'objectif de cette étude était d'estimer l'évolution, depuis 2005, de l'incidence et des caractéristiques des cas de syphilis récente dans la cohorte de patients vivant avec le VIH (PVVIH) suivis en Martinique.

Matériels et méthodes Tous les PVVIH suivis dans le service de maladies infectieuses et tropicales du CHU de Martinique entre le 01/01/2 005 et le $31 / 10 / 2015$ avec un diagnostic de syphilis récente (syphilis primaire, secondaire ou latente précoce) ont été inclus. Les caractéristiques des patients diagnostiqués entre le 01/01/2 005 et le $31 / 05 / 2010$ ( $1^{\text {re }}$ période) ont été comparées à celles des patients diagnostiqués entre le 01/06/2010 et le 31/10/2015 ( $2^{\text {nde }}$ période).

Résultats Cent quatre vingt quinze épisodes de syphilis récentes ont été diagnostiqués parmi les PVVIH suivis entre le $1^{\text {er }}$ janvier 2005 et le 31 octobre 2015. L'incidence annuelle est de $1,8 \%$ en $2005,4,3 \%$ en 2008 ( $1^{\text {er }}$ pic épidémique), $1,2 \%$ en 2013 et $3,4 \%$ en 2015 ( $2^{\text {nd }}$ pic épidémique). Le diagnostic d'infection par le VIH précède l'épisode syphilitique dans $76,4 \%$ des cas. La proportion d'HSH est significativement plus importante sur la $2^{\text {nde }}$ période que sur la $1^{\text {re }}$ période $(77,1 \%$ versus $50 \%, \mathrm{p}=0,0001)$. Quarante-cing pour cent des cas concernent des récidives de syphilis durant la $2^{\text {nde }}$ période vs $27,9 \%$ durant la $1^{\text {re }}$ période $(\mathrm{p}=0,02)$. Les paramètres immuno-virologiques (taux de CD4, charge virale VIH) sont comparables entre les 2 périodes.

Conclusion Ces données montrent une reprise importante de l'épidémie de syphilis depuis 2014 en Martinique. Contrairement au pic épidémique de 2008 pour lequel les HSH étaient minoritaires, cette reprise épidémique concerne très majoritairement les $\mathrm{HSH}$. Ces résultats incitent à renforcer les politiques de prévention des pratiques sexuelles à risque.

Aucun lien d'intérêt

\section{IST-07}

Infections disséminées à gonocoque : étude rétrospective départementale de 2009 à 2015

M. Cachera (1), M. Lagrange-Xélot (1), L. Bellec (1), M. Moiton (1), R. Manaquin (2), S. Picot (2), A. Lignereux (3), I. Degasne (1)

(1) CHU réunion Site Nord Félix Guyon, Saint-Denis,

(2) CHU Réunion Site Sud GHSR, Saint-Pierre,

(3) CH Gabriel Martin, Sainte-Paule.

Introduction Les infections disséminées à gonocoque (IDG) sont peu fréquentes. Nous avons voulu évaluer les caractéristiques des IDG à l'échelle d'un département d'outre-mer.

Matériels et méthodes $\mathrm{Il}$ s'agit d'une étude descriptive rétrospective multicentrique entre le $1^{\text {er }}$ janvier 2009 et le 30 juin 2015 . Les cas ont étérecensés en croisant les données des laboratoires d'analyses et celles des Départements d'Information Médicale des 4 hôpitaux de l'île. Les IDG ont été définies par la mise en évidence par culture sur sang ou sur liquide synovial de la bactérie Neisseria gonorrhoeae $(\mathrm{Ng})$ ou par l'existence d'un syndrome clinique faisant évoquer une IDG associé à la mise en évidence de $N g$ sur d'autres sites (culture ou PCR).

Résultats Vingt-trois patients ( 7 femmes et 16 hommes, âgés de 19 à 67 ans) ont été présenté une IDG. Le nombre de cas annuel d'IDG augmente entre 2009 et 2015 (de 0 à 6). La moyenne d'âge des patients est de 40 ans, 29 ans pour les femmes et 44 ans pour les hommes. Neuf patients ont été contaminés lors d'un voyage à l'étranger. Le diagnostic a reposé sur la mise en évidence de $N g$ sur sang ou liquide synovial dans 19 cas $(82 \%)$ et sur l'existence d'un syndrome clinique faisant évoquer une IDG associé à la mise en évidence de $\mathrm{Ng}$ sur un autre site pour 4 cas. Le délai diagnostique était de $11 \mathrm{~J}(4-30 \mathrm{j})$

Les atteintes présentées étaient: arthrites $\mathrm{n}=17(73 \%)$, ténosynovites $\mathrm{n}=6(26 \%)$, atteintes cutanées $\mathrm{n}=10(43 \%)$, endocardite $\mathrm{n}=1$, prostatite $\mathrm{n}=1$. Seuls 5 patients $(21 \%)$ ont présenté des signes génitaux.

Le diagnostic a été confirmé par culture chez 18 patients (5 sur sang, 10 sur liquide synovial, 1 sur abcès de ténosynovite, 1 sur écouvillon urétral et 1 sur ECBU) et par biologie moléculaire pour 12 patients ( 9 sur liquide synovial et 3 sur prélèvement génital). Dix souches $(43 \%)$ ont montré une résistance aux fluoroquinolones, dont 6 souches acquises à l'étranger (Madagascar et Thaïlande) et 7 aux tétracyclines. Toutes les souches étaient sensibles à la ceftriaxone et au cefixime. Les patients ont été traités par ceftriaxone dans 19 cas, par cefixime dans 2 cas, par amoxicilline-acide clavulanique dans 1 cas et par amoxicilline dans un cas. La durée moyenne de traitement était de $15 \mathrm{j}$ (4-42). Huit patients ont eu besoin d'une intervention chirurgicale. Six patients avaient des séquelles articulaires à un mois.
Conclusion Les IDG sont en augmentation dans notre département mais reste sous-évoquées. L'atteinte articulaire est quasi-constante. Le diagnostic d'IDG est à évoquer devant une mono ou poly-arthrite en particulier en cas d'association à une ténosynovite et/ou des lésions cutanées, y compris en l'absence de symptomatologie génitale.

Aucun lien d'intérêt

\section{IST-08}

Prévalence alarmante d'infections à Chlamydia trachomatis dans une CDAG-CIDDIST récente

M.-A. Khuong-Josses, P. Nivôse, C. Charpentier, B. Frison, M. Poupard, I. Gros, C. Chaplain

Hôpital Delafontaine, Saint-Denis.

Introduction Selon les données de l'INVS, le nombre d'infections urogénitales à Chlamydia trachomatis (CT) continue d'augmenter chez l'homme comme chez la femme dans les sites à participation constante les 3 dernières années, même si cette augmentation reflète en partie un accroissement des pratiques de dépistage.

Matériels et méthodes Matériel et méthodes :

Dans le cadre du rapport annuel d'activité 2015, nous avons réalisé une étude rétrospective qui décrit les données épidémiologiques et médicales des personnes ayant consulté de façon anonyme dans une Cdag (Centre de dépistage anonyme et gratuit) - Ciddist (Centre d'information de dépistage et de diagnostic des infections sexuellement transmissibles).

Résultats Sur une période d'un an, 1754 personnes ont consulté, dont 895 (392 femmes et 503 hommes) avaient les critères de dépistage d'IST définis par l'HAS.

Concernant l'âge, $54(6 \%)$ et $163(18 \%)$ avaient respectivement moins de 18 ans et 20 ans. Une infection à CT était retrouvée chez $14.25 \%$ des personnes (126/884). Cette prévalence élevée était plus marquée chez les femmes de moins de 25 ans avec 18,4\% (48/261) de prélèvements positifs. Respectivement, ces taux étaient à $8,6 \%(11 / 128), 13,6 \%(51 / 368)$ et $10,2 \%(16 / 127)$ chez les femmes de plus de 25 ans, les hommes de moins de 30 ans et les hommes de plus de 30 ans.

De façon non surprenante, $74,6 \%(50 / 67)$ des hommes et $91,5 \%(54 / 59)$ des femmes étaient asymptomatiques.

Concernant les autres infections dépistées, 30 infections à Neisseria gonorrhoeae ont été dépistées, 18 hommes $(3,6 \%)$ et 12 femmes $(3,1 \%)$, ainsi qu'11 infections à VIH sur 851 personnes prélevées $(1,3 \%)$.

Dix-huit personnes avaient une co-infection CT et Neisseria gonorrhoeae.

Les jeunes sont moins susceptibles de revenir chercher leurs résultats que les autres patients : : chez les moins de 18 ans, $82 \%$ des résultats sont retirés $(\mathrm{n}=45 / 55)$ versus $90 \%(\mathrm{n}=822 / 911)$ pour les plus de $18 \mathrm{ans}, \mathrm{OR}=3.99$, $\mathrm{p}=0.04$.

Conclusion Le taux de patients asymptomatiques est très élevé mais plutôt conforme aux données de la littérature en France. Par contre le taux d'infections à $\mathrm{CT}$ est très supérieur et particulièrement chez les jeunes femmes avec un taux de $18,4 \%$ de prélèvement positif à $\mathrm{CT}$. Ces résultats incitent à maintenir et même renforcer les mesures de dépistage et de prévention des IST chez ces patients.

Liens d'intérêts déclarés : BMS

M.-A. K.-J. Investigateur principal pour GSK. Remboursement congrès

\section{IST-09}

Intérêt d'un dépistage multi-sites de Chlamydia trachomatis (CT) et Neisseria gonorrhoeae (NG) chez des patients asymptomatiques en CIDDIST

B. Tissier (1), G. Wartel (1), B. Roquebert (1), C. Gaud (1) (1) CHU La Réunion.

Introduction L'incidence des infections par NG et CT ne cesse de progresser. Le caractère asymptomatique de certaines localisations, notamment ano-rectales et vaginales, constituent un réservoir pour la transmission. Cette étude tente d'évaluer l'intérêt d'un dépistage combiné multi-sites.

Matériels et méthodes Étude transversale monocentrique chez des patients de plus de 15 ans consultant au CIDDIST du 01/12/2014 au 01/06/2015. Les 